\title{
6-DOFs hardware-in-the-loop system for wind tunnel tests of floating offshore wind turbines
}

Preprint of OMAE2019-95967 submitted to the 38th International Conference on Ocean, Offshore \& Arctic Engineering, June 9-14, 2019, Scotland, Glasgow

\author{
A. Fontanella ${ }^{1}$, I. Bayati ${ }^{2}$, F. Taruffi ${ }^{1}$, F. La Mura ${ }^{1}$, A. Facchinetti ${ }^{1}$, M. Belloli ${ }^{1}$ \\ ${ }^{1}$ Mechanical Engineering Department, Politecnico di Milano, Milano, Via La Masa 1, \\ 20156, Italy. \\ ${ }^{2}$ Maritime Research Institute Netherlands (MARIN), Wageningen, 6708 PM, \\ The Netherlands.
}

Author contact email: alessandro.fontanella@polimil.it

\section{ABSTRACT}

This article presents a hardware-in-the-loop (HIL) methodology developed at Politecnico di Milano (PoliMi) to perform wind tunnel tests on floating offshore wind turbines (FOWTs). The 6-DOFs HIL setup is presented, focusing on the main differences with respect to a previous 2-DOFs system. Aerodynamic, rotor and control related loads, physically reproduced by the wind turbine scale model, must be measured in real-time and integrated with the platform numerical model. These forces contribute to couple wind turbine and floating platform dynamics and their correct reproduction is of fundamental importance for the correct simulation of the floating system behavior. The procedure developed to extract rotor loads from the available measurements is presented, discussing its limitations and the possible uncertainties introduced in the results. Results from verification tests in no-wind conditions are presented and analyzed to identify the main uncertainty sources and quantify their effect on the reproduction of the floating wind turbine response to combined wind and waves.

\section{2 nomenclature}

DOF Degree of freedom

EOM Equation of motion

FOWT Floating offshore wind turbine

FRF Frequency response function

HIL Hardware-in-the-loop

OL Open-loop

PoliMi Politecnico Milano

RAO Response amplitude operator 
$f_{n} \quad$ Natural frequency

$h$ Damping ratio

$\underline{F}_{a, r o t} \quad$ Aerodynamic rotor loads

$\underline{F}_{a, t w r} \quad$ Aerodynamic tower loads

$\underline{F}_{\text {corr }} \quad$ Correction loads

$\underline{F}_{\text {gyro }} \quad$ Gyroscopic loads

$\underline{F}_{m, r o t}$ Mechanical rotor loads

$\underline{F}_{R U A G} \quad$ Tower-base loads

$\underline{F}_{w t} \quad$ Wind turbine loads

$\underline{l}_{a}$ HexaFloat actuators actual position

$q$ Platform position from HIL model

$\underline{q}_{s} \quad$ Platform set-point

$\underline{q}_{a} \quad$ Platform actual position

$\underline{\tilde{q}}_{a} \quad$ Platform actual position estimate

\section{INTRODUCTION}

Scale model testing precedes and completes full-scale testing in the study and design of floating offshore wind turbines. The design of FOWTs strongly relies on mathematical models and numerical tools that are used to simulate the system response to the environmental conditions of interest. The quality of the design resulting from this process is strictly connected to the fidelity of the used simulation tools, hence validation and verification of these instruments is a critical task [28] and it must rely on high-quality data. Scale model testing provides data required to fulfill the above mentioned requirements, reducing costs, time and widening the variety of dynamic conditions that are investigated in a fully controlled environment. Wind tunnel testing of wind turbine scale models has always been a fundamental tool for the development of innovative control logics [15]. By providing the scale model of the same mechatronic capabilities of a real wind turbine, it is possible to investigate the effects of advanced controllers and observers, avoiding all the risks that should be faced in full-scale experiments.

Hardwar-in-the-loop (HIL) and software-in-the-loop (SIL) experimental techniques were recently developed in order to overcome the limitations of traditional ocean basin experiments, enhancing the possibilities offered by scale models of investigating very precise phenomena in strictly controlled and reproducible conditions. HIL/SIL ocean basin tests are generally based on a physical scale model of the floating platform and wind turbine tower combined with an actuation system that reproduces rotor loads. Measured platform displacements are the input for a rotor numerical model that is integrated in real-time to compute aerodynamic and control induced loads. These are applied on the physical scale model by means of different devices, like tendons [26, 4], a winch system [20], a ducted fan $[2,29]$ or a multifan system [6] (force control, motion feedback).

A complementary testing technique was developed at Politecnico di Milano in order to perform wind tunnel tests of FOWTs [10] exploiting the unique characteristics of the atmospheric boundary layer (ABL) test section of PoliMi wind tunnel (Galleria del Vento Politecnico di Milano, GVPM [1]). The relatively high Reynolds numbers reached operating inside a wind tunnel and the possibility to control the wind profile and inflow turbulence makes HIL wind tunnel experiments about FOWT an important tool for the study of the floating wind turbines control problem. The wind turbine control logic can be developed and verified even if in presence of unavoidable scaling imperfections, obtaining a first estimate of the loads on the main wind turbine components and evaluating the stability margins for different operating conditions. A physical scale model of the wind turbine 


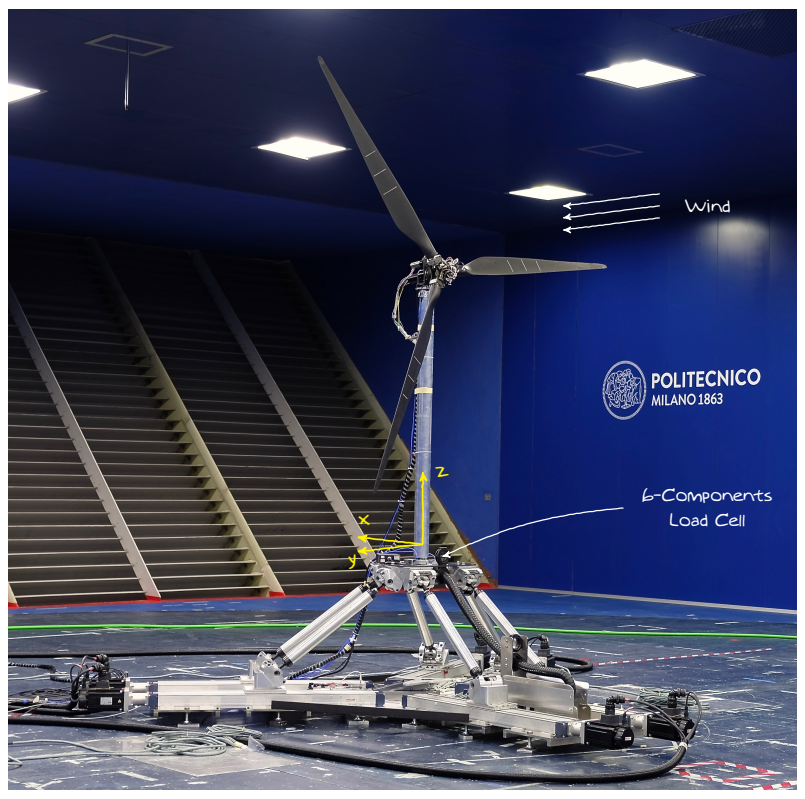

Figure 1: The HIL setup inside the atmospheric boundary layer test section of PoliMi wind tunnel.

allows to reproduce aerodynamic and rotor loads that are combined with numerically computed hydrodynamic and wave loads for the real-time integration of platform rigid-body motion equations. The resulting displacements are imposed to the wind turbine model by means of a 6-DOFs parallel kinematic robot (motion control, force feedback) [13].

The PoliMi HIL testing methodology was initially developed to simulate the dynamics of platform surge and pitch DOFs only [11]. In that work the floating system numerical model used for HIL tests is presented and verified against the FAST v8.16 model of the DeepCwind semi-submersible [25] for no-wind conditions. Then, the system robustness to real measurements and non-ideal actuation is evaluated from experimental test in still-air. In [14], 2-DOFs experiments about the DeepCwind floating system are repeated, extending no-wind verification tests and adding test cases in constant laminar wind in order to evaluate the effect of aerodynamic loads on the FOWT global response.

In this work, the 6-DOFs HIL system, currently used for wind tunnel tests is presented. The numerical model and its real-time implementation, introduced in [12], are briefly discussed to show how the different parts of the floating system are modeled in the hybrid experiment. The attention is focused on the methodology developed in order to properly extract the wind turbine tower-base loads. The latter are analyzed, investigating the nature of their main components, also from the output of specific wind tunnel tests, carried out within the framework of the European H2020 project LIFES50+. The effects that neglecting part of the wind turbine loads from the HIL force feedback has on the reproduction of the FOWT motion is carefully evaluated. Finally, results from verification tests in still-air are presented to understand the uncertainties introduced by the HIL measurement and actuation chain on the floating wind turbine response.

\section{THE HIL METHODOLOGY}

The methodology for wind tunnel HIL tests developed in [11] for the 2-DOFs (surge and pitch) case was extended to 6-DOFs (surge, sway, heave, roll, pitch and yaw) in [12]. The main equations at the base of the HIL numerical model are recalled here for the sake of clarity. 
The 6-DOFs platform response to wind and wave loads is computed from real-time integration of Eq. 1 that describes the floating system rigid-body dynamics.

$$
\left(\left[M_{s}\right]+\left[A_{\infty}\right]\right) \underline{\ddot{q}}+\left[R_{s}\right] \underline{\dot{q}}+\left[K_{s}\right] \underline{q}=\underline{F}_{p l a t}+\underline{F}_{w t}
$$

where $\left[M_{s}\right]$ is the floating system (platform and wind turbine) mass matrix about the 6 platform DOFs, $\left[A_{\infty}\right]$ is the infinite-frequency added mass matrix, resulting from 3D panel code (e.g. WAMIT) computations, $\left[R_{s}\right]$ is an optionally added linear damping matrix and $\left[K_{s}\right]$ the gravitational and buoyancy stiffness matrix of the floating system. The complete expressions of the above mentioned matrices are reported in the Appendix of [12]. EOMs of Eq. 1 are integrated in real-time for a forcing term that is the sum of platform loads $\underline{F}_{p l a t}$ and wind turbine loads $\underline{F}_{w t}$.

\section{Platform loads}

Floating platform loads are the sum of different contributions summarized in Eq. 2.

$$
\underline{F}_{\text {plat }}=\underline{F}_{r a d}+\underline{F}_{w e}+\underline{F}_{v i s c}+\underline{F}_{m o o r}
$$

Radiation loads $\underline{F}_{r a d}$, modeling the memory effect, are implemented exploiting the state-space approximation $[17,16]$ of the convolution integral between platform velocities and retardation matrix (impulse response). The first and second order wave exciting forces $\underline{F}_{w e}$ are implemented as precomputed time histories stored in multidimensional lookup tables. These are obtained combining the wave spectrum and the complex, frequency-dependent transfer functions between wave elevation and platform loads, resulting from panel code pre-simulations. Newmann approximation is used to introduce second order diffraction loads. Offline calculation of wave forces is preferred above real-time calculation since it is associated with a lower computational cost. Viscous forces $\underline{F}_{\text {visc }}$ are computed from solution of Morison's equation, keeping into account wave kinematics as detailed in [12]. Loads exerted by the mooring system on the floating platform $\underline{F}_{\text {moor }}$ are obtained integrating, simultaneously with the platform model [12], a lumped-mass model of the catenary lines. The latter was derived from the validated formulation at the base of the FAST/MoorDyn module [21], introducing some simplifications to make it compatible with the real-time constraints, though not introducing relevant differences in the overall dynamics, as assessed in [12].

\section{Wind turbine loads}

The reproduction of loads exerted by the wind turbine on the floating platform relies on the physical wind turbine scale model. In particular, wind turbine loads $\underline{F}_{w t}$ are extracted according to Eq. 3 from 6-components load cell (RUAG 192-6I) measurements. The sensor is mounted at tower base (see Fig. 1) and the loads reduction point is coincident with the (ideal) point where the wind turbine tower is connected to the floating platform.

$$
\underline{F}_{w t}=\underline{F}_{R U A G}-\underline{F}_{c o r r}
$$

Wind turbine loads $\underline{F}_{w t}$ are obtained subtracting from the overall loads $\underline{F}_{R U A G}$ the inertial and gravitational components associated with the wind turbine scale model $\underline{F}_{\text {corr }}$. The reproduction of the inertial and gravitational wind turbine loads is entirely demanded to the numerical model since the wind turbine model inertial properties (mass and mass distribution) do not exactly match the scaled values [9]. Moreover, being the experiment governed by non-Froude scaling laws, a gravity acceleration different from $9.81 \mathrm{~m} / \mathrm{s}^{2}$ would be required in order to physically reproduce weight loads. 


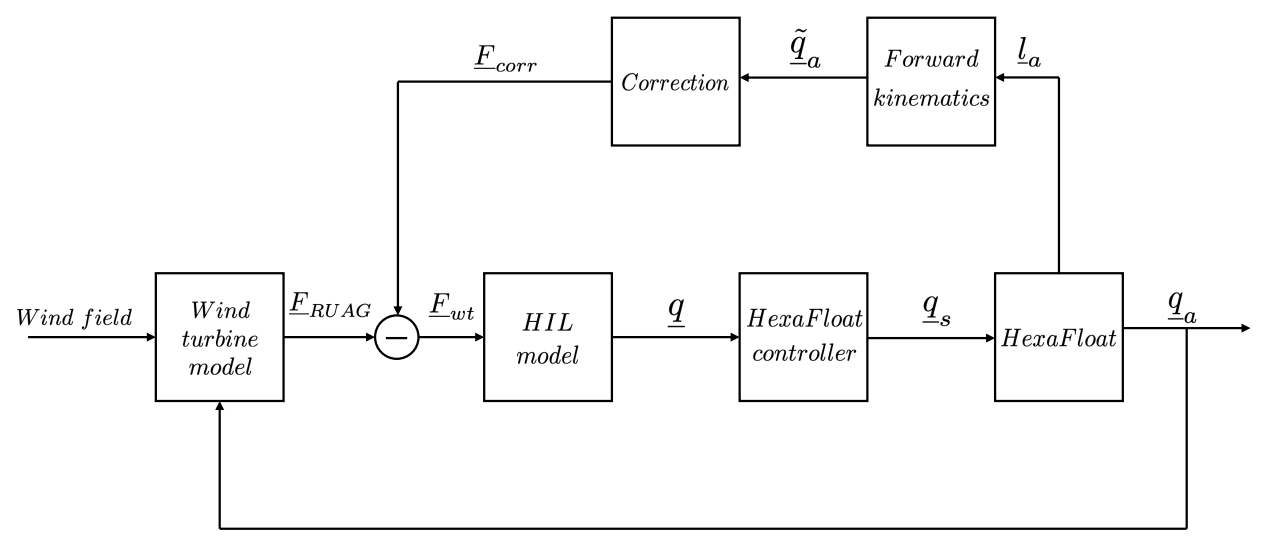

Figure 2: Experimental setup control scheme.

The procedure required to computed the so-called correction loads $\underline{F}_{\text {corr }}$ is going to be discussed in the following sections.

Wind turbine loads $\underline{F}_{w t}$ are given by the superposition of different force contributions, as expressed by Eq. 4.

$$
\underline{F}_{w t}=\underline{F}_{a, r o t}+\underline{F}_{a, t w r}+\underline{F}_{g y r o}+\underline{F}_{m, r o t}
$$

In particular, $\underline{F}_{w t}$ include the effect of aerodynamic rotor loads $\underline{F}_{a, r o t}$, aerodynamic tower loads $\underline{F}_{a, t w r}$, gyroscopic moments $\underline{F}_{g y r o}$ and mechanical rotor loads $\underline{F}_{m, r o t}$. The 6 components of $\underline{F}_{a, r o t}$ are given by thrust and non-thrust aerodynamic loads. In presence of a non-null rotor tilt angle, the projection of thrust force according to the tower-base reference frame (where wind turbine loads are measured) results in a surge force, heave force and pitch moment. Aerodynamic sway and heave forces, pitch and yaw moments are caused by a non-uniform wind speed across the rotor area, usually due to turbulence and wind shear. Finally, the aerodynamic rotor torque is associated with a towerbase roll moment. Aerodynamic tower loads $\underline{F}_{a, t w r}$ are due to tower drag that results, when the wind turbine tilt is different from zero, in a surge force, heave force and pitch moment. Gyroscopic pitch and yaw moments $\underline{F}_{\text {gyro }}$ are caused by a variation of the orientation of the wind turbine rotor rotation axis caused by platform yaw and pitch motion, respectively. Mechanical rotor loads $\underline{F}_{m, r o t}$ are due to rotor inertia and unbalanced mass distribution.

\section{EXPERIMENTAL SETUP CONTROL SCHEME}

The HIL control system is described by the block diagram of Fig. 2. The wind turbine scale model (physical subsystem) is operated in the ABL test section of PoliMi wind tunnel and it is exposed to a properly generated wind field.

Load cell measurements $\underline{F}_{R U A G}$ are sent to the HIL controller where these are low-pass filtered and processed according to Eq. 3 to extract wind turbine loads. The obtained wind turbine loads are used for real-time integration of Eq. 1 that results in the position of the tower connection point to the floating platform $q$. This represents the set-point for the HexaFloat robot, which is converted into the HexaFloat reference frame $\underline{q_{s}}$ (robot's actuation and kinematics) and then physically provided as $\underline{q}_{a}$.

The experimental setup described above allows two operational configurations, identified as open-loop and hardware-in-the-loop (HIL). In the first case, the floating system numerical model is integrated in 
real-time excluding the force feedback from the force transducer at tower base. In this way the wind turbine scale model is moved in feed-forward, according to behavior of the numerical substructure only, neglecting the contribution of wind turbine loads. In the second case, the floating system model is integrated keeping into account the force feedback $\underline{F}_{w t}$.

\section{Correction loads}

Correction loads $\underline{F}_{\text {corr }}$ are computed in order to extract wind turbine loads from tower-base measurements, as described by Eq. 3. The wind turbine model can be described as a single rigid-body moving in space if the wind and wave loads frequency is below the first natural frequency of its main components. In that case, the inertial and gravitational loads resulting from the wind turbine model motion, and then correction loads $\underline{F}_{c o r r}$, are expressed by Eq. 5 .

$$
\underline{F}_{\text {corr }}=\left[M_{t}\right] \underline{\tilde{q}}_{a}+\left[K_{t}\right] \underline{\tilde{q}}_{a}
$$

where $\left[M_{t}\right]$ and $\left[K_{t}\right]$ are the 6 -by- 6 mass and gravitational stiffness matrices of the wind turbine model with respect to the platform DOFs. The process of real-time estimating $\underline{F}_{c o r r}$ requires the knowledge of the wind turbine model position and acceleration $\left(\underline{q}_{s}, \underline{\ddot{q}}_{s}\right)$ and its inertial and geometrical properties. The HexaFloat controller is featured by high and low-level algorithms that modify the set-points $q$ generated by HIL controller in order to optimize the machine performance and safety (e.g. control readiness and acceleration limits) [24, 23]. The motion imposed to the wind turbine model $\underline{q}_{s}$ is thus slightly different from references coming from the integration of Eq. 1 . The actual position of the six HexaFloat motors $\underline{l}_{a}$ is used to reconstruct the wind turbine model position $\underline{q}_{a}$ by solving non-linear forward kinematic equations by means of a Jacobian free monotonic descent (JFMD) algorithm [27]. The optimum $\left[M_{t}\right]$ and $\left[K_{t}\right]$ parameters were found starting from the physical linearized terms expressed in [12]. A custom genetic algorithm was run offline, trying to minimize wind turbine loads $\underline{F}_{w t}$ from time series acquired in still-air in an open-loop configuration.

\section{LIMITED FORCE FEEDBACK}

The HIL methodology presented in this work was conceived as a tool to investigate the effect of unsteady aerodynamic thrust loads and rotor torque on the floating system performance.

Surge and pitch components of wind turbine loads $\underline{F}_{w t}$ are mostly given by aerodynamic thrust and aerodynamic tower loads. The correct reproduction of aerodynamic thrust loads is ensured by the performance scaling procedure adopted to design the wind turbine model rotor [8]. Similarly, towerdrag loads $\underline{F}_{a, t w r}$ are correctly reproduced by properly choosing the wind turbine tower diameter. When performing tests aimed at assessing the along-wind FOWT dynamics, the above mentioned aerodynamic load components represent a large fraction of the corresponding $\underline{F}_{R U A G}$ components, and are predominant on the inertial and gravitational loads associated with the wind turbine scale model. This, together with the fact that the inertial and geometrical parameters required to define the corresponding correction loads $\underline{F}_{\text {corr }}$ are easily estimated, facilitates the extraction of the surge and pitch aerodynamic loads from tower-base measurements.

The reproduction of other wind turbine forces with the HIL methodology discussed in the present work is generally more difficult. The sway and heave components of $\underline{F}_{w t}$ are due to non-thrust aerodynamic rotor loads and mechanical loads caused by rotor imbalance. The vertical projection of tower drag also contributes to the heave force. Non-thrust aerodynamic loads are small when performing tests in laminar flow, since the wind speed is almost constant across the rotor area. The heave component of tower drag is also small, since the wind turbine tilt is usually limited to small 
angles. These aerodynamic load components are of the same order of magnitude of residual loads associated with the wind turbine model inertia and weight, that cannot be completely removed due to the non-perfect force subtraction of Eq. 3. It is therefore very difficult to have a correct estimate of the wind turbine heave and sway forces.

The roll component of $\underline{F}_{w t}$ is due to rotor inertial and aerodynamic loads. The yaw component of $\underline{F}_{w t}$ is due to the gyroscopic moment caused by the pitch motion and the aerodynamic moment caused by an uneven wind speed across the rotor. While the second contribution is negligible in laminar flow, the first one is significative but difficult to reproduce, since the wind turbine scale model rotor inertia usually exceeds the scaled target value (correctly scaling the rotor inertia would require very light blades, difficult to be manufactured in presence of stiffness constraints [8]). The extraction of roll and yaw moments from tower-base loads is hindered by the estimation of the parameters of $\left[M_{t}\right]$ required for correction loads computation.

As explained above, the sway, heave, roll and yaw components of $\underline{F}_{w t}$ are difficult to reproduce in HIL tests. Errors in load contributions used for the numerical model integration (force feedback) result in an incorrect prediction of platform dynamics also leading to unphysical or even unstable behaviors. Tests with wind that are going to be presented in a following section also demonstrated that these load components are negligible. For these reasons, and being wind tunnel tests mainly focused on the along-wind FOWT dynamics that is predominant, it was preferred to set to zero the sway, heave, roll and yaw components of $\underline{F}_{w t}$. Still, the motion in the other directions that may arise from waves excitation and mooring lines dynamics is kept and applied to the wind turbine model. Thus, HIL experiments fully account for the effects of such motions on the FOWT behavior.

\section{VERIFICATION TESTS}

Experimental tests were performed in order to assess the effectiveness of the HIL methodology presented above. All results are upscaled according to the scale factors defined for the experiment and presented at full-scale. Limitations on the wind turbine force feedback were assessed studying the nature of $\underline{F}_{w t}$ loads measured for different wind conditions through free-decay tests in open-loop configuration. A series of still-air tests allowed to verify the floating platform dynamics and quantify the uncertainties introduced in the HIL methodology on the reproduction of the rigid-body FOWT motions. HIL results are compared to the output of corresponding open-loop tests, where the FOWT motion depends on the floating system numerical model only. The performance of the latter (in a stand-alone configuration) was already evaluated and validated in [12], where it was compared to an equivalent higher-order FAST model. Thus, differences between HIL and open-loop experiments do not depend on modeling choices adopted for the platform numerical model, since these are mainly related to the uncertainties affecting the procedure used to extract wind turbine loads, in terms of parameters estimation and motion measurement.

\section{Reference floating system}

Verification tests were performed for a reference floating system formed by the OO-Star Wind Floater [22] coupled with the DTU $10 \mathrm{MW}$ reference wind turbine [5]. The physical wind turbine scale model adopted for the experiments was the 1/75 model of the DTU 10MW RWT designed and built at PoliMi within the EU H2020 LIFES50+ project $[9,7]$. Tests were performed in non-Froude scaled conditions [13], combining a length scale factor of $1 / 75$ with a velocity scale factor of $1 / 3$. Scale factors for all the other dimensions were obtained from dimensional analysis. 

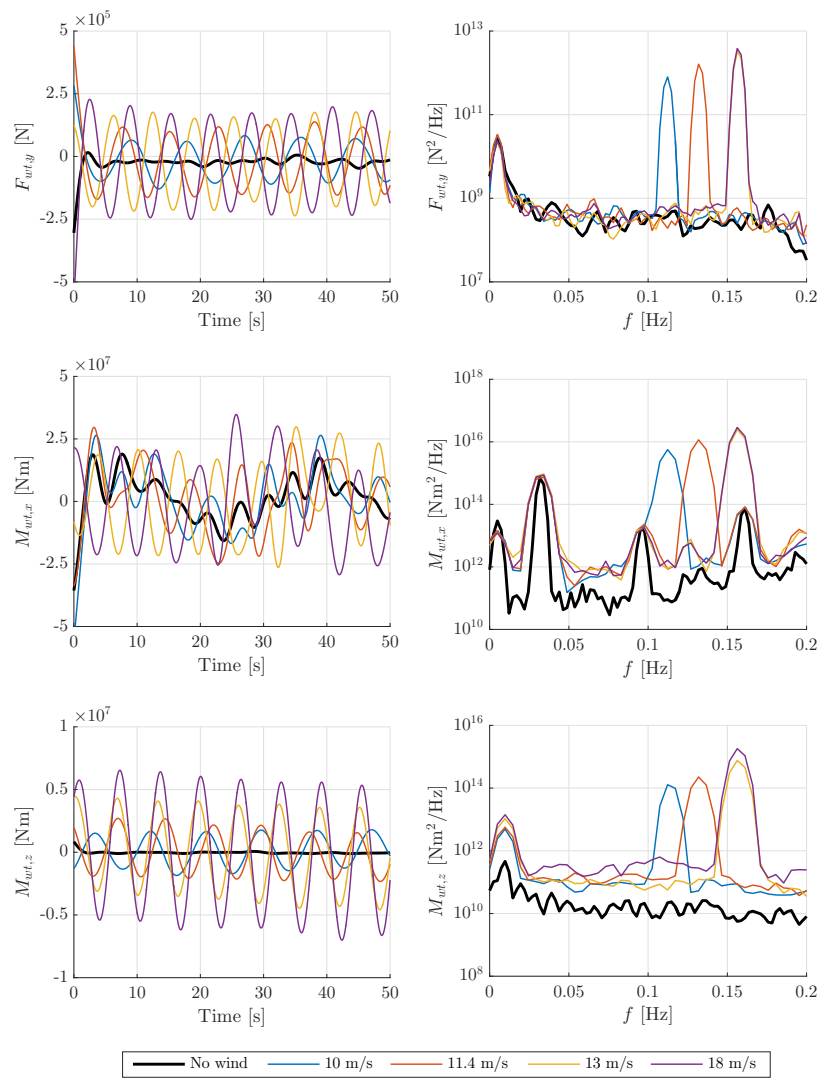

Figure 3: Time history and PSD of the sway, roll and yaw wind turbine load components for different free-decay tests and wind turbine operating conditions.

\section{Assessment of limited force feedback}

The effects that excluding some wind turbine force components has on the reproduction of FOWT dynamics is discussed in [3] making use of numerical simulations, with specific reference to ocean basin HIL tests. Specific experimental tests aimed at assessing the nature of the sway, roll and yaw $\underline{F}_{w t}$ components were performed in order to complement the main findings of the above mentioned work.

Decay tests were performed in open-loop configuration, alternatively imposing a $8 \mathrm{~m}$ initial displacement and $3.5^{\circ}$ rotation to the sway, roll and yaw DOFs, in order to generate significative non-thrust wind turbine loads. Tests were repeated in still-air and for four different mean wind speeds, corresponding to an equal number of wind turbine operating conditions. The action of the wind turbine scale model variable-speed variable-pitch (VS-VP) controller $[18,19]$ was enabled in all the test runs. Wind turbine loads were computed in real-time from tower-base measurements, according to the procedure described by Eq. 3-5, but the floating system numerical model integration was performed excluding force feedback.

The time history and PSD of wind turbine sway, roll and yaw load components from sway, roll and yaw free-decay tests respectively, are shown in Fig. 3. The sway force, roll and yaw moments show a significative harmonic component at the floating system motion frequency that is combined, when 

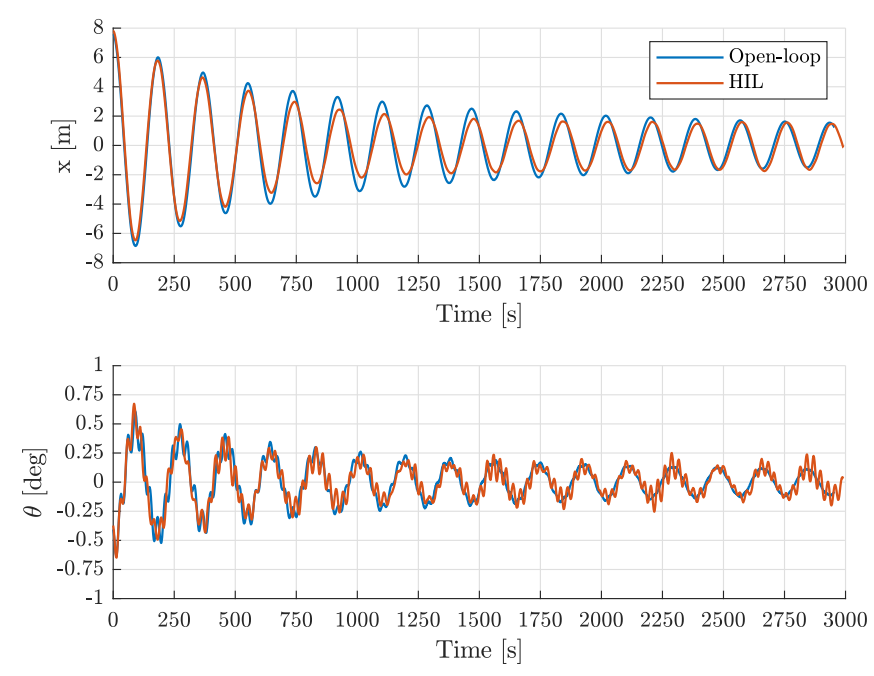

Figure 4: Platform surge and pitch free-decay response in open-loop and HIL configuration for an initial surge displacement of $8 \mathrm{~m}$.

Table 1: Platform surge and pitch dynamic properties in open-loop and HIL configuration evaluated from a free-decay test (initial surge displacement of $8 \mathrm{~m}$ ).

\begin{tabular}{lcccc}
\hline & \multicolumn{2}{c}{ Surge } & \multicolumn{2}{c}{ Pitch } \\
Type & $f_{n}[\mathrm{~Hz}]$ & $h[\%]$ & $f_{n}[\mathrm{~Hz}]$ & $h[\%]$ \\
\hline OL & 0.0054 & 0.0188 & 0.0323 & 0.0013 \\
HIL & 0.0053 & 0.0208 & 0.0365 & 0.0152 \\
\hline
\end{tabular}

the wind turbine is operating, with a second harmonic component at the rotor angular frequency. The low-frequency harmonic component shows a very weak dependence on wind speed conditions. Its amplitude is almost constant passing from still-air to $18 \mathrm{~m} / \mathrm{s}$ wind speed and significative variations are seen only for $M_{w t, z}$. Differences are instead seen for the high-frequency component, which amplitude generally increases with rotor speed, reaching a constant value in above-rated conditions.

\section{Free-decay tests}

Free-decay tests were performed in order to quantify the uncertainties introduced by the HIL methodology in the reproduction of the linear dynamic properties of the floating platform rigid-body motion modes, and to verify the effectiveness of the force subtraction procedure for the extraction of $\underline{F}_{w t}$. Experiments were performed in open-loop and HIL configurations by imposing to a single platform DOF a perturbation with respect to the static equilibrium position and allowing the system to move freely. The dynamic properties of the selected rigid-body motion modes were derived from the analysis of the first 5 oscillation cycles at the mode frequency, extracted from the selected mode response by applying a variable width band-pass filter (e.g. in order to compute the pitch mode dynamic properties, the pitch mode response was isolated from the platform pitch response time series). The motion frequency was detected from the FFT of the analyzed platform DOF response, whereas damping data were obtained applying the logarithmic decrement method. Results about the surge and pitch DOFs only are reported here, being these two DOFs directly affected by the HIL force feedback. 

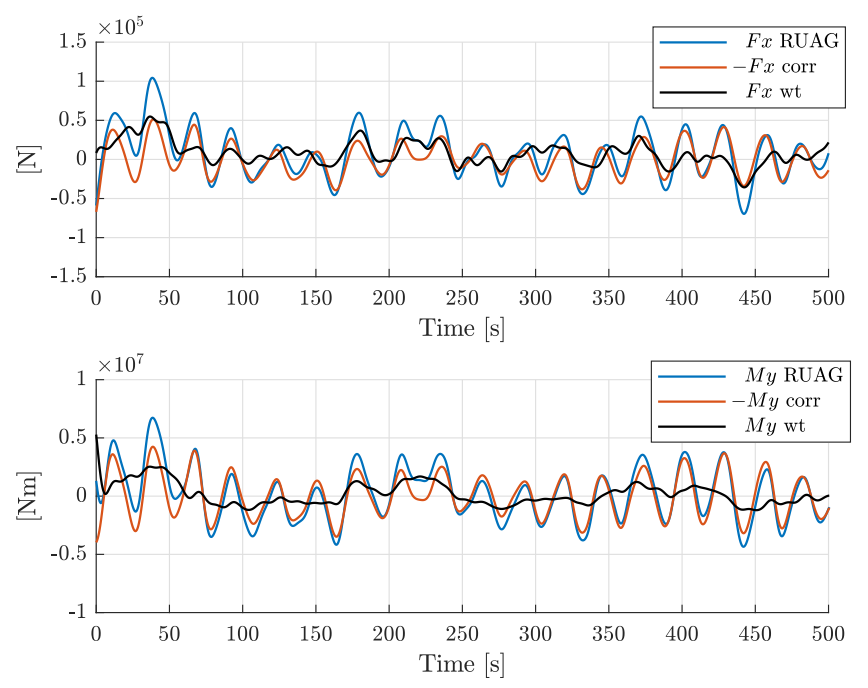

Figure 5: Tower-base surge forces and pitch moments for a free-decay test (initial surge displacement of $8 \mathrm{~m}$ ).

The surge and pitch response for an initial surge displacement of $8 \mathrm{~m}$ is shown in Fig. 4. As visible, the response measured in HIL configuration is in good agreement with the corresponding results for the open-loop case. The main difference is seen for the pitch DOF, which response is induced by coupling with the surge DOF. Considering the HIL configuration, in the first response cycles, pitch oscillations at the pitch mode frequency are more damped than what is obtained when operating in open-loop. However, after some time, the amplitude of the pitch mode response is amplified to be damped some cycles later. This cyclic behavior of the pitch mode is absent from the open-loop response and is introduced by the HIL force feedback.

The dynamic properties of the two platform rigid-body motion modes computed from the time histories presented in Fig. 4 are resumed in Tab. 1. As seen the differences introduced by the force feedback (HIL configuration) are quite small and limited to the pitch mode damping. The time histories of the different load contributions, measured by the tower-base (RUAG) sensor, correction loads (with opposite sign) and wind turbine loads fed to the HIL model, for the surge decay of Fig. 4 are shown in Fig. 5. The estimated correction loads are adherent to the measured surge force and pitch moment and the resulting wind turbine loads fed to the HIL model are almost null. Residual inertial and gravitational loads are due to uncertainties in the force subtraction procedure, mainly related to parameters estimation and motion measurement. This is advisable in still-air conditions where inertial and gravitational wind turbine loads are prevailing on the other load components.

The surge and pitch response for an initial pitch rotation of $3.5^{\circ}$ is shown in Fig. 6. Some differences are seen between the open-loop and HIL response of both the considered platform DOFs and these are more marked for the pitch DOF. As highlighted by the dynamic properties of Tab. 2, the surge mode shows an increased frequency and lower damping in HIL configuration than in open-loop. A small frequency variation is seen also for the pitch mode which damping is also significantly increased. The time histories of the different loads used for HIL force feedback are shown in Fig. 7. Also in this case correction loads are very close to the force and moment measured at tower base. The magnitude of the resulting wind turbine loads are small. 

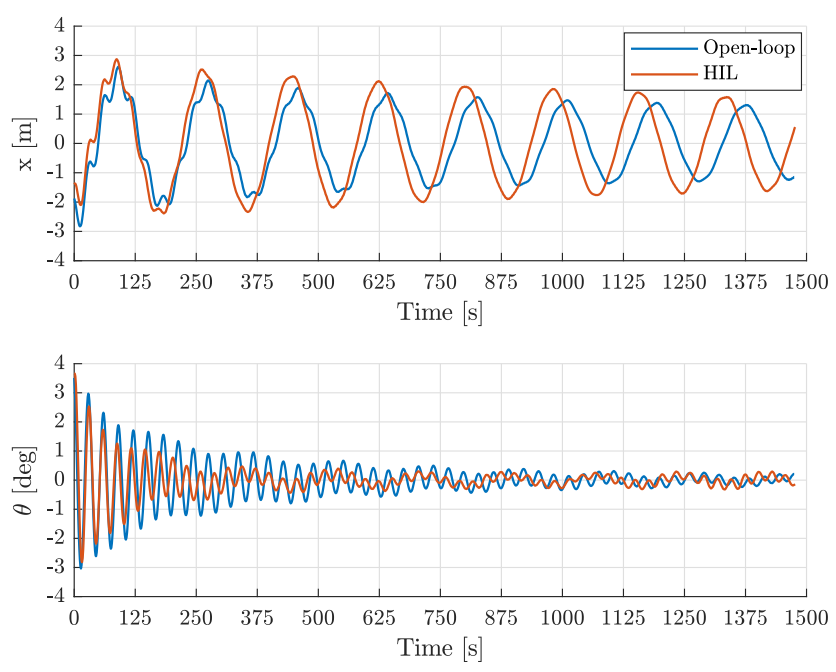

Figure 6: Platform surge and pitch free-decay response in open-loop and HIL configuration for an initial pitch rotation of $3.5^{\circ}$.

Table 2: Platform surge and pitch dynamic properties in open-loop and HIL configuration evaluated from a free-decay test (initial pitch rotation of $3.5^{\circ}$ ).

\begin{tabular}{lcccc}
\hline & \multicolumn{2}{c}{ Surge } & \multicolumn{2}{c}{ Pitch } \\
Type & $f_{n}[\mathrm{~Hz}]$ & $h[\%]$ & $f_{n}[\mathrm{~Hz}]$ & $h[\%]$ \\
\hline OL & 0.0053 & 0.0099 & 0.0326 & 0.0186 \\
HIL & 0.0054 & 0.0089 & 0.0355 & 0.0303 \\
\hline
\end{tabular}

\section{Pink noise tests}

Pink noise waves from $0^{\circ}$ (frequency range $0.003-0.05 \mathrm{~Hz}$ ) were run in still-air in order to assess the floating platform DOFs non-linear response to broad-band hydrodynamic loads. In particular, the excitation provided by wave loads allowed to identify the frequency response function (FRF) between wave height and the platform DOFs response. From this, it was possible to highlight how the HIL measurement and actuation chains affect the reproduction of floating system dynamics at different frequencies and, in particular, in the low frequency range where platform modes are found.

The FRF between wave height and platform surge, heave and pitch, coincident with the platform RAO for the selected DOFs, is shown in Fig. 8 for the open-loop and HIL configurations. The analysis is limited to three DOFs being the response of the others (sway, roll and yaw) negligible for $0^{\circ}$ waves. As visible, the platform dynamics as reproduced by the HIL system are very close to what is obtained in open-loop. The main differences are found for the pitch DOF that shows a slightly higher frequency and damping in HIL configuration, in agreement with the results of free-decay tests. The response of the heave DOF is slightly less damped for the HIL system. Being the heave force component excluded from the force feedback, this difference is due to the coupling with the other DOFs, that are affected by the measured wind turbine loads. 

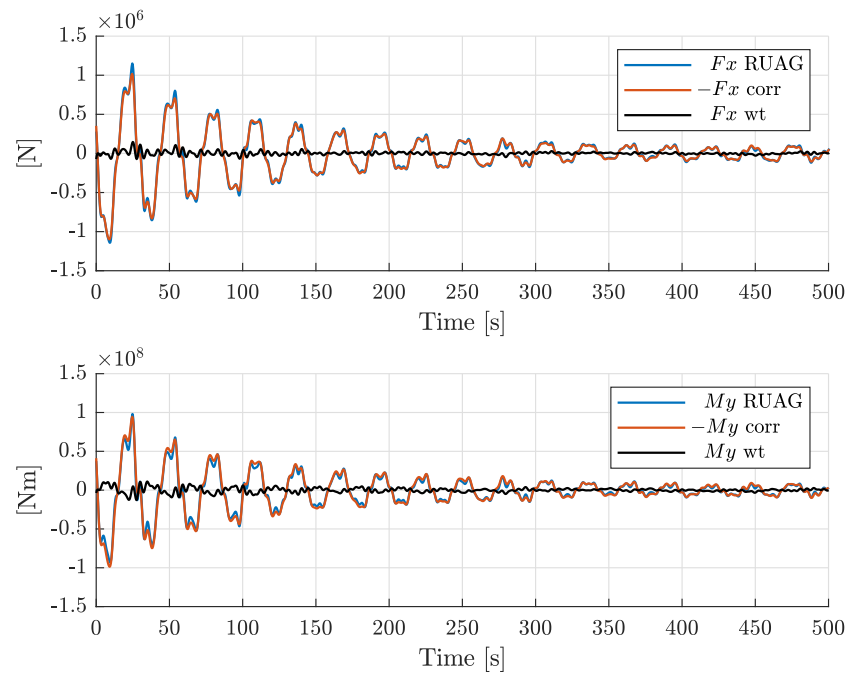

Figure 7: Tower-base surge forces and pitch moments for a free-decay test (initial pitch rotation of $\left.3.5^{\circ}\right)$.

\section{CONCLUSIONS}

This paper presented the 6-DOFs HIL methodology developed at PoliMi to investigate the global dynamics of floating offshore wind turbines inside a wind tunnel. The measurement chain that allows to extract wind turbine loads from the tower-base load cell measurements was deeply discussed. In particular, the nature of wind turbine loads in along-wind cases was studied and it was found that thrust-related forces are the most important for the reproduction of the FOWT response. The sway, heave, roll and yaw components of wind turbine loads were neglected since small if compared to thrust-related forces and of the same order of magnitude of the corresponding residual inertial and gravitational loads that are not completely removed in the force subtraction procedure. At the same time, the sway, heave, roll and yaw motions induced by other excitation sources are reproduced and their effects on the thrust-related wind turbine loads is correctly accounted for.

The uncertainties introduced by the HIL measurement and actuation chains in the platform DOFs response were assessed through still-air experimental tests. Free-decay tests and $0^{\circ}$ pink noise waves confirmed the implementation choices, evidencing the HIL system capability of correctly reproducing the dynamic properties of the FOWT rigid-body motion modes, in particular surge and pitch.

Further tests are advisable for assessing the capability of correctly modeling the other DOFs dynamics, especially for load cases with significative wave misalignment. Improvements could also be achieved employing more complex HIL control schemes, with additional control loops on the wind turbine model position setpoint. This would lead to a more general formulation of the current HIL methodology, that could be adopted for other types of hybrid wind tunnel tests [30] where, differently from FOWT, it is not possible to identify predominant system dynamics.

\section{ACKNOWLEDGEMENTS}

This project has received funding from the European Union's Horizon 2020 research and innovation program under grant agreement No 640741. The content of the work does not report the opinion of the European Commission and reflects only the views of the author(s), including errors or omissions. 

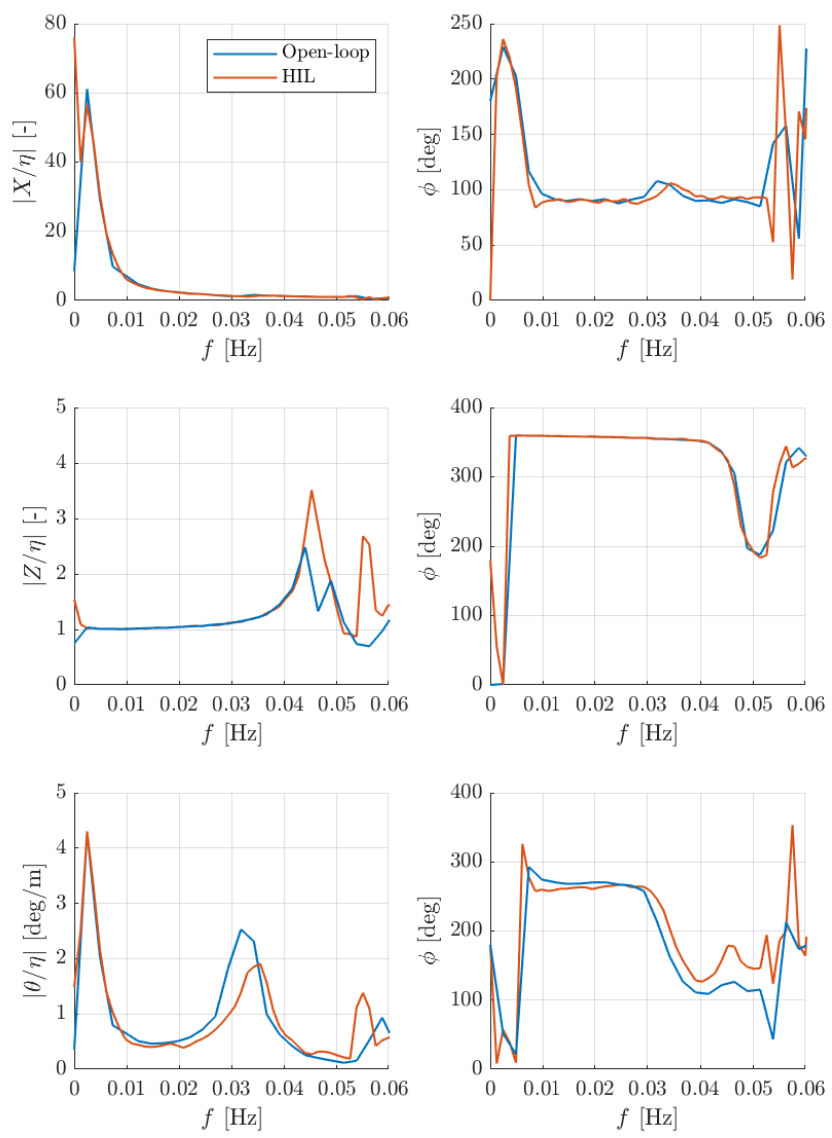

Figure 8: RAO (amplitude left, phase right) for platform surge, heave and pitch DOFs in open-loop and HIL configuration.

The European Commission is also not liable for any use that may be made of the information contained herein.

\section{References}

[1] Galleria del Vento Politecnico di Milano. http://www.windtunnel.polimi.it.

[2] J. Azcona, F. Bouchotrouch, M. Gonzalez, J. Garciandia, X. Munduate, F. Kelberlau, and T. A. Nygaard. Aerodynamic Thrust Modelling in Wave Tank Tests of Offshore Floating Wind Turbines Using a Ducted Fan. Journal of Physics: Conference Series, 524(1):012089, 2014.

[3] E. E. Bachynski, V. Chabaud, and T. Sauder. Real-time hybrid model testing of floating wind turbines: sensitivity to limited actuation. Energy Procedia, 80:2 - 12, 2015. doi: 10.1016/j.egypro.2015.11.400.

[4] E. E. Bachynski, M. Thys, T. Sauder, V. Chabaud, and L. O. Sæther. Real-time hybrid model testing of a braceless semi-submersible wind turbine. part II: Experimental results. 35th International Conference on Offshore Mechanics and Arctic Engineering (OMAE) - Busan, Korea, 6(49972), 2016. doi: 10.1115/OMAE2016-54437.

[5] C. Bak, F. Zahle, R. Bitsche, K. Taeseong, A. Yde, L. C. Henriksen, M. H. Hansen, J. P. A. A. 
Jose, M. Gaunaa, and A. Natarajan. The DTU 10-MW Reference Wind Turbine. DTU Wind Energy Report, 2013.

[6] T. Battistella, D. De Los Dolores Paradinas, A. Meseguer Urban, and R. Guanche Garcia. High Fidelity Simulation of Multi-MW Rotor Aerodynamics by Using a Multifan. 37th International Conference on Offshore Mechanics and Arctic Engineering (OMAE) - Madrid, Spain, 10(51319), 2018. doi: 10.1115/OMAE2018-77606.

[7] I. Bayati, M. Belloli, L. Bernini, E. Fiore, H. Giberti, and A. Zasso. On the functional design of the DTU $10 \mathrm{MW}$ wind turbine scale model of LIFES50+ project. Journal of Physics: Conference Series, 753(2), 2016. http://stacks.iop.org/1742-6596/753/i=2/a=022028.

[8] I. Bayati, M. Belloli, L. Bernini, and A. Zasso. Aerodynamic design methodology for wind tunnel tests of wind turbine rotors. Journal of Wind Engineering and Industrial Aerodynamics, 167:217 - 227, 2017. doi: 10.1016/j.jweia.2017.05.004.

[9] I. Bayati, M. Belloli, L. Bernini, and A. Zasso. Scale model technology for floating offshore wind turbines. IET Renewable Power Generation, 11(9):1120 - 1126, 2017. doi: 10.1049/ietrpg.2016.0956.

[10] I. Bayati, M. Belloli, D. Ferrari, F. Fossati, and H. Giberti. Design of a 6-DoF Robotic Platform for Wind Tunnel Tests of Floating Wind Turbines. Energy Procedia, 53:313 - 323, 2014. doi: 10.1016/j.egypro.2014.07.240.

[11] I. Bayati, A. Facchinetti, and M. Belloli. Wind tunnel 2-DOF hybrid/HIL tests on the OC5 floating offshore wind turbine. 36th International Conference on Offshore Mechanics and Arctic Engineering (OMAE) - Trondheim, Norway, 10(57786), 2017. doi: 10.1115/OMAE2017-61763.

[12] I. Bayati, A. Facchinetti, A. Fontanella, and M. Belloli. 6-DoF hydrodynamic modelling for wind tunnel hybrid/HIL tests of FOWT: the real-time challenge. 37th International Conference on Offshore Mechanics and Arctic Engineering (OMAE) - Madrid, Spain, 10(51319), 2018.

[13] I. Bayati, A. Facchinetti, A. Fontanella, H. Giberti, and M. Belloli. A wind tunnel/HIL setup for integrated tests of Floating Offshore Wind Turbines. Journal of Physics: Conference Series, 1037(5):052025, 2018. http://stacks.iop.org/1742-6596/1037/i=5/a=052025.

[14] I. Bayati, A. Facchinetti, A. Fontanella, F. Taruffi, and M. Belloli. Analysis of FOWT dynamics in 2-DOF hybrid HIL wind tunnel experiments. Ocean Engineering.

forthcoming.

[15] C. L. Bottasso, F. Campagnolo, and V. Petrovic. Wind tunnel testing of scaled wind turbine models: Beyond aerodynamics. Journal of Wind Engineering and Industrial Aerodynamics, 127:11 - 28, 2014. doi: 10.1016/j.jweia.2014.01.009.

[16] T. Duarte. SS Fitting, Theory and User Manual.

[17] T. Duarte, M. Alves, J. Jonkman, and A. Sarmento. State-space realization of the waveradiation force within FAST. 32nd International Conference on Offshore Mechanics and Arctic Engineering (OMAE) - Nantes, France, 8(55423), 2013. doi: 10.1115/OMAE2013-10375.

[18] A. Fontanella, I. Bayati, and M. Belloli. Control of Floating Offshore Wind Turbines: ReducedOrder Modeling and Real-Time Implementation for Wind Tunnel Tests. 37th International Conference on Offshore Mechanics and Arctic Engineering (OMAE) - Madrid, Spain, 10(51319), 2018. 10.1115/OMAE2018-77840. 
[19] A. Fontanella, F. Taruffi, I. Bayati, and M. Belloli. Variable-speed Variable-pitch control for a wind turbine scale model. EERA DeepWind 2018, 15th Deep Sea Offshore Wind R\&D Conference, forthcoming.

[20] S. Gueydon, R. Lindeboom, W. van Kampen, and E. J. de Ridder. Comparison of two Wind Turbine Loading Emulation Techniques Based on Tests of a TLP-FOWT in Combined Wind, Waves and Current. ASME 2018 1st International Offshore Wind Technical Conference (IOWTC) San Francisco, CA, 2018. doi: 10.1115/IOWTC2018-1068.

[21] M. Hall and A. Goupee. Validation of a lumped-mass mooring line model with DeepCwind semisubmersible model test data. Ocean Engineering, 104:590-603, 2015. doi: 10.1016/j.oceaneng.2015.05.035.

[22] K. Muller, F. Lemmer, and W. Yu. Deliverable D4.2 Public Definition of Two LIFES50+ 10MW Floater Concepts. 2013.

[23] F. La Mura, P. Romano, E. Fiore, and H. Giberti. Workspace Limiting Strategy for 6 DOF Force Controlled PKMs Manipulating High Inertia Objects. Robotics, 7(1), 2018. doi: $10.3390 /$ robotics 7010010 .

[24] F. La Mura, G. Todeschini, and H. Giberti. High Performance Motion-Planner Architecture for Hardware-In-the-Loop System Based on Position-Based-Admittance-Control. Robotics, 7 , 2018. doi: $10.3390 /$ robotics 7010008 .

[25] A. Robertson, J. Jonkman, F. Wendt, A. Goupee, and H. Dagher. Definition of the OC5 DeepCwind Semisubmersible Floating System. Technical Report, National Renewable Energy Laboratory, 2014. NREL/TP-5000-60601.

[26] T. Sauder, V. Chabaud, M. Thys, E. E. Bachynski, and L. O. Sæther. Real-Time Hybrid Model Testing of a Braceless Semi-submersible Wind Turbine. Part I: the Hybrid Approach. 35th International Conference on Offshore Mechanics and Arctic Engineering (OMAE) - Busan, Korea, 6(49972), 2016. doi: 10.1115/OMAE2016-54435.

[27] G. Shen, Y. Tang, J. Zhao, H. Lu, X. Li, and G. Li. Jacobian free monotonic descent algorithm for forward kinematics of spatial parallel manipulator. Advances in Mechanical Engineering, 8, 2016. doi: $10.1177 / 1687814016645447$.

[28] G. A. M. van Kuik, J. Peinke, R. Nijssen, D. Lekou, J. Mann, J. N. Sørensen, C. Ferreira, J. W. van Wingerden, D. Schlipf, P. Gebraad, H. Polinder, A. Abrahamsen, G. J. W. van Bussel, J. D. Sørensen, P. Tavner, C. L. Bottasso, M. Muskulus, D. Matha, H. J. Lindemboom, S. Degraer, O. Kramer, S. Lenhoff, M. Sonneschein, P. E. Sørensen, R. W. Kunneke, P. E. Morthorst, and $\mathrm{K}$. Skytte. Long-term research challenges in wind energy - a research agenda by the European Academy of Wind Energy. Wind energy science, pages $26-27,2016$.

[29] F. Vittori, F. Bouchotrouch, F. Lemmer, and J. Azcona. Hybrid Scaled Testing of a 5MW Floating Wind Turbine Using the SiL Method Compared With Numerical Models. 37th International Conference on Offshore Mechanics and Arctic Engineering (OMAE) - Madrid, Spain, 10(51319), 2018. doi: 10.1115/OMAE2018-77853.

[30] A. Zasso, T. Argentini, I. Bayati, M. Belloli, and D. Rocchi. Super-long bridges with floating towers: The role of multi-box decks and Hardware-In-the-Loop technology for wind tunnel tests. IOP Conference Series: Materials Science and Engineering, 276(1), 2017. http://stacks.iop.org/1757-899X/276/i=1/a =012008. 